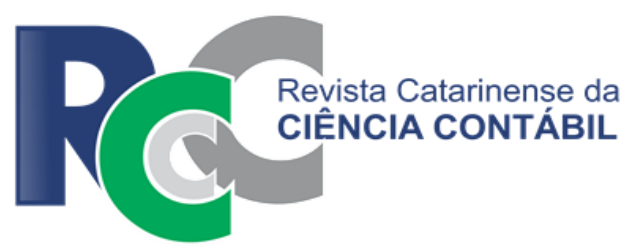

\title{
EXCESSO DE CONFIANÇA DO CHIEF EXECUTIVE OFFICER E A PRÁTICA DE GERENCIAMENTO DE RESULTADOS
}

\section{OVERCONFIDENT CHIEF EXECUTIVE OFFICER AND EARNINGS MANAGEMENT PRACTICE}

\author{
CRISTIAN BAU DAL MAGRO \\ Doutor em Ciências Contábeis e Administração na Universidade \\ Regional de Blumenau (FURB). Endereço: Servidão Anjo da Guarda, \\ 295-D / Efapi | 89809-900 / Chapecó/SC | Brasil. \\ E-mail: cristianbaumagro@gmail.com
}

\section{MARCELLO CHRISTIANO GORLA}

Doutor em Ciências Contábeis e Administração na Universidade Regional de Blumenau (FURB). Endereço: Rua Antônio da Veiga, 140 | Itoupava Seca | 89030-903 / Blumenau/SC / Brasil.

E-mail: gorlacontabil@yahoo.com.br

\section{ROBERTO CARLOS KLANN}

Doutor em Ciências Contábeis e Administração pela Universidade Regional de Blumenau (FURB). Professor do Programação de PósGraduação em Ciências Contábeis da FURB. Endereço: Rua Antônio da Veiga, 140 / Itoupava Seca / 89030-903 / Blumenau/SC / Brasil.

E-mail: rklann@furb.br

\section{RESUMO}

Diversos estudos têm sugerido que o excesso de confiança leva a expectativas de desempenho futuro, e os gerentes podem desenvolver predisposição para o gerenciamento de resultados. $O$ objetivo do estudo é identificar a influência do excesso de confiança de gestores nas práticas de gerenciamento de resultados das empresas brasileiras. A metodologia da pesquisa é descritiva, com abordagem quantitativa, por meio de pesquisa documental. Os métodos estatísticos utilizados foram: entropia, TOPSIS e a regressão linear múltipla. A amostra foi composta de 127 empresas brasileiras listadas na BM\&FBovespa. Conclui-se que o menor excesso de confiança impacta positivamente no gerenciamento de resultados. Sugere que os gestores com menor confiança acreditam, com menor intensidade, em suas habilidades, e, para garantir resultados organizacionais que satisfaçam às expectativas de analistas, praticam o gerenciamento de resultados de maneira oportuna para aumentar os resultados organizacionais.

Palavras-Chave: Excesso de confiança. Gerenciamento de resultados. Teoria comportamental.

\section{ABSTRACT}

Several studies have suggested that overconfidence leads to expectations of future performance, and managers can develop predisposition to earnings management. The objective of the study is to identify the influence of overconfident managers on Brazilian companies' earning management practices. The methodology of the research is descriptive, with quantitative approach, through documentary research. The statistical methods used were: entropy, TOPSIS and multiple linear regression. The sample consisted of 127 Brazilian companies listed on the BM\&FBovespa. It is 
concluded that lower overconfidence has a positive impact on earnings management, suggesting that less confident managers believe with less intensity in their abilities, and, in order to guarantee organizational results that satisfy the expectations of analysts, they conduct the earnings management in an opportunistic way so as to increase organizational results.

Keywords: Overconfident. Earnings management. Behavioral theory.

\section{INTRODUÇÃO}

O excesso de confiança tem sido argumentado, por comportamentalistas, como um fator importante para o mercado financeiro. Pode afetar o comportamento humano nas diversas decisões organizacionais (De Bondt \& Thaler,1995). Nesse sentido, a teoria comportamental foi utilizada para explicar a existência de vieses, no comportamento dos agentes, que podem gerar a tomada de decisões pautada em aspectos irracionais e, dessa forma, interferir na estruturação do capital organizacional (Thaler, 1999).

Estudos recentes têm demonstrado que os aspectos irracionais do comportamento humano afetam as decisões operacionais de investimento e financiamento, bem como o comportamento discricionário sobre as escolhas contábeis. O excesso de confiança pode gerar comportamentos enviesados que são capazes de gerar, por parte dos gestores, crenças sobre habilidades que estão acima do curso normal no mundo dos negócios (Weinstein, 1980; Baker, Ruback \& Wurgler, 2004). Portanto, o ser humano pode superestimar suas capacidades, acreditando que seu poder de decisão é mais assertivo que o de outros (Svenson, 1981; Taylor \& Brown, 1988; Alicke, Klotz, Breitenbecher, Yurak \& Vredenburg, 1995).

Os gestores que possuem tendência de superioridade sobre suas habilidades, julgamentos e previsões futuras, possuem características que expressam o excesso de confiança (Barber \& Odean, 2001; Simon \& Houghton, 2003; Malmendier \& Tate, 2005; Dushnitsky, 2010). Os gestores com essas características tendem a superestimar suas capacidades, para afetar os resultados, subestimar os eventos aleatórios e demonstrar maior propensão para as previsões tendenciosamente otimistas (Hribar \& Yang, 2015). Os fatores psicológicos, características pessoais e racionalidade limitada podem ser utilizados para explicar os comportamentos oportunistas, e, assim, ajudar a elucidar possíveis distorções gerenciais que ocorrem nas organizações (Lin, Ho \& Chen, 2005).

Os resultados reportados pelas organizações, nos demonstrativos contábeis, podem decorrer de vieses comportamentais oportunistas dos Chief Executive Officer (CEOs). Tal fator advém das escolhas contábeis de natureza discricionária, que não possuem relação com a realidade econômica do negócio. O comportamento oportunista, na discricionariedade das escolhas contábeis, pode ser motivado pelas influências exógenas da firma e pelo comportamento dos indivíduos, os quais desvirtuam executivos em direção as práticas de gerenciamento de resultados (Martinez, 2008).

O excesso de confiança dos gestores pode acarretar decisões que envolvem escolhas contábeis de maior ou menor discricionariedade. $O$ efeito do excesso de confiança na qualidade da informação contábil, sob a proxy do gerenciamento de resultados, foi examinado em dois estudos com empresas dos Estados Unidos da América (EUA). Hribar e Yang (2015) argumentam que o excesso de confiança fez executivos (CEOs) anunciarem previsões de lucros com base em expectativas excessivas de resultados futuros. Schrand e Zechman (2012) sugerem que as empresas gerenciadas por CEOs que possuem excesso de confiança podem exercer, de maneira acidental e/ou intencional, o gerenciamento de resultados.

O estudo de Li e Hung (2013), com empresas do Taiwan, sugere que o excesso de confiança aumenta o comportamento oportunista dos gestores. Pode acarretar no uso mais intensificado das práticas de gerenciamento de resultados com vistas a aumentar os lucros. Estudos empíricos sugerem que o excesso de confiança aumenta a expectativa de desempenho futuro, e os gerentes com tais características possuem maior propensão para a prática de gerenciamento de resultados, com finalidade de satisfazer as expectativas dos analistas (Malmendier \& Tate, 2005; Hribar \& Yang, 2015; Schrand \& Zechman, 2012; Li \& Hung, 2013). 
Diante do exposto, criou-se a seguinte problemática: Qual a influência do excesso de confiança de gestores no gerenciamento de resultados das empresas brasileiras listadas na BM\&FBovespa? O objetivo é identificar a influência do excesso de confiança de gestores no gerenciamento de resultados das empresas brasileiras listadas na BM\&FBovespa.

O estudo busca acrescentar, na literatura da qualidade da informação contábil, vieses comportamentais que podem afetar as decisões de executivos corporativos sobre as escolhas contábeis que visam à distorção, dentro das normas do reporte da informação contábil. A literatura empírica sobre gerenciamento de resultados tem examinado os incentivos econômicos, pessoais e corporativos. Contudo, faz-se necessário adicionar os fatores comportamentais nos incentivos para a prática de gerenciamento de resultados.

Há escassez de pesquisas que adicionam evidências da psicologia individual sobre as escolhas contábeis oportunistas (Hribar \& Yang, 2015). De acordo com Martinez (2013), existe a necessidade de integrar os aspectos comportamentais de agentes, gestores e auditores com as práticas oportunidades que envolvem o gerenciamento de resultados. A contribuição do estudo está vinculada à lacuna teórica da literatura da qualidade da informação contábil. Adiciona evidências do comportamento individual do ser humano sobre práticas oportunistas.

No campo prático, a pesquisa oferece indícios de que os fatores comportamentais devem ser observados na avaliação da qualidade da informação contábil. Portanto, na avaliação dos resultados reportados pelas firmas, é preciso que os analistas, investidores e demais stakeholders observem as atitudes dos gestores, principalmente buscando segregar as empresas gerenciadas por aqueles com comportamentos excessivamente confiantes daqueles que possuem mais cautela nas ações e atitudes organizacionais.

A pesquisa diferencia-se dos estudos de Hribar e Yang (2015), Schrand e Zechman (2012) e de Li e Hung (2013), por apresentar diferentes métricas de mensuração do excesso de confiança, aliadas ao oportunismo nas escolhas contábeis. Os estudos anteriores foram realizados em países desenvolvidos com fortes características no mercado acionário e, portanto, a realização de um estudo com empresas brasileiras pode acrescentar perspectivas contraditórias sobre o efeito do excesso de confiança na qualidade da informação contábil.

\section{REFERENCIAL TEÓRICO}

\subsection{Aspectos comportamentais do excesso de confiança dos gestores}

Heaton (2002) e Malmendier e Tate (2005) apontam que os gestores com excesso de confiança tendem a valorizar em demasia os retornos futuros dos projetos de investimento. Outros estudos têm documentado que o excesso de confiança afeta os investimentos, financiamentos e as políticas de dividendos das organizações (Malmendier \& Tate, 2008; Deshmukh, Goel \& Howe, 2013; Malmendier, Tate \& Yan, 2011).

A literatura aponta para a existência de, ao menos, três tipos de excesso de confiança. Conforme Hilary e Hsu (2011), o primeiro está presente quando os indivíduos, muitas vezes, exageram na sua própria capacidade e avaliam seus atributos de gestão, decisão, capacidade e responsabilidade, como sendo melhores dos que os dos outros. O excesso de confiança não é visto apenas na área de finanças e contabilidade. Peterson (2007) relata, por exemplo, que 70\% das pessoas acreditam ser melhores motoristas que os outros.

O segundo envolve as crenças extremas relativas a um objetivo padrão. Por exemplo, estima-se que ocorre um evento com $90 \%$ de probabilidade, quando, na verdade, é preciso estimar uma probabilidade inferior. Por fim, o terceiro relaciona-se ao efeito da ponderação, o qual envolve a relação entre informações particulares e públicas (Hilary \& Hsu, 2011). Nesse aspecto, os indivíduos excessivamente confiantes acreditam que sua informação privada possui maior precisão do que realmente tem, e, portanto, atribuem a ela um maior peso.

Esta pesquisa posiciona-se no atributo de excesso de confiança estabelecido por Hilary e Hsu (2011), em que os gestores subestimam sua capacidade de gestão nas decisões organizações. A literatura sobre excesso de confiança, no processo de tomada de decisão, tem apresentado evidências empíricas relacionadas ao comportamento excessivamente confiante de empresários, conforme Cooper, Woo e Dunkelberg (1988), em relação aos executivos, por Russo 
e Schoemaker (1992), e aos gerentes, por Dittrich, Güth e Maciejovsky (2005). A pesquisa estabelece avanço empírico nos estudos de excesso de confiança. Incrementa uma visão sobre as escolhas contábeis que afetam a qualidade da informação contábil.

\subsection{Excesso de confiança dos gestores e o gerenciamento de resultados}

Para Watts e Zimmerman (1990), o gerenciamento de resultados pode ser característico das práticas oportunistas, pela discricionariedade, para seu benefício ex post com efeitos redistributivos da riqueza entre as partes de um contrato. O gerenciamento de resultados contábeis encontra refúgio na flexibilidade das normas e regulamentos, que permitem alternativas distintas para a contabilização de um mesmo evento (Matsumoto \& Pereira, 2009).

Santos e Grateron (2003) comentam que a expressão earnings management pode ser entendida como o gerenciamento ou manuseio dos resultados, com a intenção de demonstrar uma imagem diferente (melhor ou pior), de acordo com os diversos interesses.

Adicionalmente, os fatores psicológicos devem ser considerados na análise do comportamento dos gestores sobre as decisões organizacionais, remetendo-se também ao reporte dos demonstrativos financeiros. O excesso de confiança tem sido visto, por comportamentalistas, como um fator importante do comportamento humano de gestores no reporte de informações para o mercado acionário (Ko \& Huang, 2007).

Hribar e Yang (2015) examinaram o impacto do excesso de confiança no aumento da probabilidade de emissão, excessivamente otimista, para o gerenciamento de resultados. Os CEOs foram classificados com base no número de vezes que foram descritos como confiantes, em relação ao número de vezes que foram descritos como prudentes, cautelosos, conservadores, práticos e/ou frugais. A amostra compreendeu 640 empresas listadas na Fortune 500 . Os resultados sugerem que o excesso de confiança aumenta o viés otimista nas previsões voluntárias. Levam, pois, ao maior gerenciamento de resultados.

Li e Hung (2013) investigaram o efeito moderador do controle familiar na relação entre o excesso de confiança e o gerenciamento de resultados. Utilizaram-se de uma amostra composta por empresas listadas no mercado acionário do Taiwan. A premissa estabelece que os gestores excessivamente confiantes são propensos a se envolver em comportamentos de gerenciamento de resultados, e o controle familiar modera negativamente a relação positiva entre o excesso de confiança e o gerenciamento de resultados.

O estudo de Hsieh, Bedard e Johnstone (2014) analisou a relação entre os CEOs excessivamente confiantes e o gerenciamento de resultados, baseado em atividades reais, e também sobre as metas para atender às previsões dos analistas. $O$ excesso de confiança dos CEOs foi determinado pelo atributo das ações em tesouraria/recompra de ações. Os resultados sugerem que, anteriormente à Lei Sarbanes-Oxley (SOX) de 2002, as empresas com CEOs confiantes teriam maior propensão para as práticas oportunistas de gerenciamento de resultados pelas atividades reais. Posteriormente à Lei SOX, os resultados indicaram que os CEOs confiantes são mais propensos a utilizarem as acumulações discricionárias para 0 gerenciamento de resultados. Os resultados são consistentes com os CEOs confiantes, sentindo menor constrangimento pela aplicação da Lei SOX, visto que o gerenciamento de resultados pelas acumulações discricionários tem maior probabilidade de ser descoberto.

\subsection{Construção das hipóteses}

O estudo de Banerjee, Humphery-Jenner e Nanda (2014) fornece evidências de que as decisões dos CEOs excessivamente confiantes são divergentes, em momentos distintos à aplicação da Lei SOX. Argumentam que as empresas com CEOs confiantes tomam decisões arriscadas que distorcem os investimentos. Sugerem que as restrições de regulamentação impostas pela SOX têm sido eficazes na redução do comportamento oportunista dos CEOs que apresentam tendências para o excesso de confiança.

Os resultados do estudo de Banerjee et al. (2014) demonstram que a Lei SOX foi efetiva na redução de comportamentos oportunistas para o gerenciamento de resultados. Contudo, esclarecem que os CEOs confiantes, provavelmente, podem continuar explorando as oportunidades em determinadas práticas de gerenciamento de resultados. 
De acordo com os argumentos de Malmendier e Tate (2005), Jin e Kothari (2008), Hribar e Yang (2015), Li e Hung (2013), Hsieh et al. (2014), Banerjee et al. (2014), tem-se a premissa de possível impacto do excesso de confiança dos gestores sobre a prática de gerenciamento de resultados mediante escolhas contábeis, possibilitando argumentos para a construção da hipótese geral que norteia o estudo:

Hipótese 1: Os gestores com excesso de confiança são mais propensos a se envolver em práticas oportunistas de gerenciamento de resultados para aumentar os lucros.

Com base em pesquisas comportamentais, tem-se que o excesso de confiança pode ser mensurado a partir de dados secundários advindos de proxies anteriormente utilizadas, como: volume de ações em tesouraria, volume de dividendos pagos, alavancagem financeira e empresas familiares. Estudos fundamentam proxies de atributos psicológicos que podem ser utilizados para a mensuração do excesso de confiança dos gestores.

Estudos têm demonstrado que as empresas gerenciadas por CEOs fundadores possuem comportamento diferente daquelas gerenciadas por CEOs profissionais (Dalton \& Daily, 2001; Nelson, 2003). Além disso, consideram que os empresários possuem maior excesso de confiança que os não empresários, ou seja, empresas cujos gestores são membros da família/fundadores possuem maior excesso de confiança que aquelas geridas por profissionais contratados (Lowe \& Ziedonis, 2006; Hmieleski \& Baron, 2009; Landier \& Thesmar, 2009). Anderson e Reeb (2003) sugerem que o comportamento dos CEOs da família difere dos CEOs não familiares devido à concorrência no mercado de trabalho e aos planos de remuneração, e que, portanto, esses fatores podem determinar o excesso de confiança na tomada das decisões.

Lee, Hwang e Chen (2014) fornecem evidências de que os CEOs fundadores são mais confiantes do que as contrapartes (CEOs profissionais). Os resultados sugerem que os CEOs fundadores utilizam uma linguagem mais substancialmente otimista em suas declarações durante teleconferências de apresentação dos resultados. Os CEOs fundadores elaboram previsões de lucros maiores que os CEOs contratados. Diante das argumentações, sugere-se que empresas com controle familiar têm representantes/gestores que possuem maior excesso de confiança, em comparação com aquelas de controle profissional.

A conjectura de que o controle familiar pode moderar os efeitos do excesso de confiança sobre o gerenciamento de resultados ocorre pelas seguintes razões: a riqueza da família está ligada ao valor da empresa (Anderson \& Reeb, 2003); os membros da família estão preocupados com a reputação da empresa (Miller, Breton-Miller \& Scholnick, 2008) e com as perspectivas de longo prazo para os negócios. Esperam transferir seus ativos para as gerações futuras (GómezMejía et al., 2007). Portanto, tem-se a primeira sub-hipótese do estudo:

Hipótese 1a: Há uma relação positiva entre o controle familiar e as práticas oportunistas de gerenciamento de resultados para aumentar os lucros.

A próxima variável utilizada para mensurar o excesso de confiança dos gestores relaciona-se ao pagamento de dividendos. Ben-David, Graham e Harvey (2007) apontam que as empresas com CFOs, excessivamente confiantes, possuem menor propensão para o pagamento de dividendos, maior propensão a se envolverem em timing de mercado e maior propensão a emitirem divulgações voluntárias. Ben-David et al. (2007) consideram que as empresas geridas por CFOs otimistas apresentam maior volume de investimentos, menor volume de pagamento de dividendos e maior nível de alavancagem.

Deshmukh et al. (2013) desenvolveram um modelo de interação dinâmica entre o excesso de confiança do CEO e a política de dividendos. O modelo demonstrou que um CEO demasiadamente confiante vê o financiamento externo tão caro, que constrói uma folga financeira para as necessidades futuras de investimentos; porém, a folga financeira é construída pela redução no volume de pagamento de dividendos. Assim, o nível de pagamento de dividendos é mais baixo em empresas geridas por CEOs com maior excesso de confiança. A literatura estabelece que o menor nível de pagamento de dividendos é linear com a presença de gestores que possuem excesso de confiança. Estabeleceu-se a segunda sub-hipótese: 
Hipótese 1b: Há uma relação negativa entre o pagamento de dividendos e as práticas oportunistas de gerenciamento de resultados para aumentar os lucros.

Ben-David et al. (2007) e Deshmukh et al. (2013) determinam que empresas geridas por CEOs com excesso de confiança possuem maior nível de alavancagem financeira. Do mesmo modo, Barros e Silveira (2009) investigaram os determinantes da estrutura de capital introduzindo a perspectiva comportamental. A predição central de trabalho foi de que empresas geridas por indivíduos excessivamente confiantes serão mais endividadas que as demais. Oferecem indícios de que o excesso de confiança dos gestores pode determinar a estrutura de capital das empresas, porque tanto nas formulações estáticas quanto nas dinâmicas o coeficiente estimado para o excesso de confiança foi positivo e geralmente significante em relação à alavancagem. Desse modo, as empresas com maior alavancam financeira apresentariam características de terem gestores excessivamente confiantes, e, portanto, teriam tendência para as práticas oportunistas de gerenciamento de resultados, determinando a terceira sub-hipótese:

Hipótese $1_{c}$ : Há uma relação positiva entre a alavancagem financeira e as práticas oportunistas de gerenciamento de resultados para aumentar os lucros.

Por fim, em relação à variável recompra de ações (ações em tesouraria), Andriosopoulos, Andriosopoulos e Hoque (2013) demonstraram que a divulgação de informações e o excesso de confiança dos CEOs são significativamente determinantes no nível de recompra de ações. Sugerem que existe uma clara relação entre a divulgação de informações, o excesso de confiança dos CEOs e as taxas de conclusão da recompra de ações.

Hsieh et al. (2014) utilizaram o volume de ações em tesouraria como proxy para determinar os CEOs com excesso de confiança. Diante dos estudos apresentados, há argumentos de que as empresas com maior volume de ações em tesouraria teriam CEOs excessivamente confiantes, visto que mantêm ações ou recompra de ações pela confiança que detêm sobre resultados futuros, que remetem à maior valorização das próprias ações da companhia. Estabelece-se a quarta sub-hipótese do estudo:

Hipótese 1d: Há uma relação positiva entre o volume de ações em tesouraria e as práticas oportunistas de gerenciamento de resultados para aumentar os lucros.

A Tabela 1 mostra a construção teórica das hipóteses previamente apresentadas.

Tabela 1

\section{Construção teórica das hipóteses}

\begin{tabular}{|c|c|c|c|}
\hline $\begin{array}{c}\text { Pressupostos das variáveis de } \\
\text { excesso de confiança }\end{array}$ & $\begin{array}{l}\text { Pressupostos do } \\
\text { Excesso de } \\
\text { Confiança }\end{array}$ & Hipóteses & Base Teórica \\
\hline $\begin{array}{l}\text { Entropia/TOPSIS das variáveis } \\
\text { Empresa familiar; pagamento de } \\
\text { dividendos; alavancagem contábil } \\
\text { e ações em tesouraria }\end{array}$ & $\begin{array}{l}\text { +Excesso de } \\
\text { Confiança }\end{array}$ & $\begin{array}{c}\text { + Gerenciamento de } \\
\text { Resultados } \\
H_{1}\end{array}$ & $\begin{array}{c}\text { Malmendier e Tate (2005); Bhandari e } \\
\text { Deaves (2006); Malmendier e Tate (2008); } \\
\text { Wong (2008); Jin e Kothari (2008); Schrand } \\
\text { e Zechman (2012); Hribar e Yang (2015); Li } \\
\text { e Hung (2013); Banerjee et al. (2014); } \\
\text { Hsieh et al. (2014). }\end{array}$ \\
\hline + Empresa Familiar & $\begin{array}{l}+ \text { Excesso de } \\
\text { Confiança }\end{array}$ & $\begin{array}{l}\text { + Gerenciamento de } \\
\text { Resultados } \\
H_{1 a}\end{array}$ & $\begin{array}{c}\text { Anderson e Reeb (2003); Lowe e Ziedonis } \\
\text { (2006); Landier e Thesmar (2009); } \\
\text { Hmieleski e Baron (2009); Lee et al. (2014). }\end{array}$ \\
\hline - Pagamento Dividendos & $\begin{array}{l}+ \text { Excesso de } \\
\text { Confiança }\end{array}$ & $\begin{array}{l}\text { + Gerenciamento de } \\
\text { Resultados } \\
H_{1 b} \\
\end{array}$ & $\begin{array}{l}\text { Ben-David et al. (2007); Deshmukh et al. } \\
\text { (2013) }\end{array}$ \\
\hline + Alavancagem Contábil & $\begin{array}{l}+ \text { Excesso de } \\
\text { Confiança }\end{array}$ & $\begin{array}{l}\text { + Gerenciamento de } \\
\text { resultados } \\
H_{1 c}\end{array}$ & $\begin{array}{l}\text { Ben-David et al. (2007); Barros e Silveira } \\
\text { (2009); Deshmukh et al. (2013). }\end{array}$ \\
\hline + Ações em Tesouraria & $\begin{array}{l}+ \text { Excesso de } \\
\text { Confiança }\end{array}$ & $\begin{array}{c}\text { + Gerenciamento de } \\
\text { Resultados } \\
H_{1 d} \\
\end{array}$ & $\begin{array}{l}\text { Heaton (2002); Malmendier e Tate (2005); } \\
\text { Andriosopoulos et al. (2013); Ahmed e } \\
\text { Duellman (2013); Hsieh et al. (2014). }\end{array}$ \\
\hline
\end{tabular}

Nota. Fonte: Elaborada pelos autores. 
Observa-se na Tabela 1 que o maior volume de ações em tesouraria é caracterizado nas empresas com gestores que possuem maior excesso de confiança. Os gestores com maior excesso de confiança (medido pelo maior volume de ações em tesouraria) apresentam tendências para a maior intensidade de utilização do gerenciamento de resultados. O menor pagamento de dividendos é caracterizado nas empresas com gestores que possuem maior excesso de confiança. E, por fim, a maior alavancagem contábil é caracterizada nas empresas em que os gestores possuem maior excesso de confiança, conforme apresentado na literatura.

\section{PROCEDIMENTOS METODOLÓGICOS}

Para atingir o objetivo do estudo realizou-se uma pesquisa descritiva, com abordagem quantitativa, por meio de pesquisa documental.

\subsection{População e Amostra da Pesquisa}

A população da pesquisa compreendeu as companhias abertas brasileiras com dados disponíveis na base de dados Economatica ${ }^{\circledR}$. A amostra foi delineada considerando as empresas que dispunham das informações necessárias para operacionalização das variáveis. Primeiramente, foram excluídas as empresas que não continham informações para o modelo de regressão, que evidencia à variável dependente gerenciamento de resultados. Posteriormente, foram excluídas as empresas que não continham informações para cálculo das variáveis independentes. A amostra final da pesquisa foi composta de 127 empresas no ano de 2014 .

\subsection{Procedimentos de coleta e análise dos dados}

Os dados coletados são relacionados às variáveis utilizadas para operacionalizar o estudo, e estão expostas na Tabela 2.

Tabela 2

\section{Composição das variáveis do estudo}

\begin{tabular}{|c|c|c|c|}
\hline Variáveis & Descrição & Métrica & Sustentação teórica \\
\hline Dependente & $\begin{array}{l}\text { Gerenciamento de } \\
\text { Resultados (Accruals } \\
\text { Discricionários) }\end{array}$ & Modelo KS Quadro 3 & Modelo KS (1995) \\
\hline \multirow{5}{*}{ Independentes } & $\begin{array}{l}\text { Ações em Tesouraria } \\
\text { (recompra de ações) }\end{array}$ & $\begin{array}{c}\text { Logaritmo natural do volume de } \\
\text { ações em tesouraria da } \\
\text { empresa i no ano } t \text {. }\end{array}$ & $\begin{array}{l}\text { Andriosopoulos et al. (2013); } \\
\text { Ahmed e Duellman (2013) }\end{array}$ \\
\hline & $\begin{array}{l}\text { Volume de pagamento } \\
\text { de dividendos }\end{array}$ & $\begin{array}{c}\text { Logaritmo natural do volume de } \\
\text { pagamento de dividendos da } \\
\text { empresa i no ano } t \text {. }\end{array}$ & $\begin{array}{c}\text { Ben-David et al. (2007); Deshmukh } \\
\text { et al. (2013) }\end{array}$ \\
\hline & $\begin{array}{l}\text { Alavancagem } \\
\text { Financeira }\end{array}$ & $\frac{\text { Passivo Total }}{\text { Ativo Total }}$ & $\begin{array}{l}\text { Barros e Silveira (2009); Ben-David } \\
\text { et al. (2007) }\end{array}$ \\
\hline & Empresa Familiar & $\begin{array}{c}\text { Variável dummy igual a } 1 \text { para } \\
\text { as empresas de controle e } \\
\text { gestão familiar, e } 0 \text { caso } \\
\text { contrário. }\end{array}$ & $\begin{array}{c}\text { Anderson e Reeb (2003); Lowe e } \\
\text { Ziedonis (2006); Landier e } \\
\text { Thesmar (2009); Hmieleski e Baron } \\
\text { (2009); Lee et al. (2014) }\end{array}$ \\
\hline & $\begin{array}{l}\text { Ranking do Excesso } \\
\text { de Confiança }\end{array}$ & $\begin{array}{l}\text { Ranking (índice) que determina } \\
\text { o excesso de confiança de } \\
\text { cada organização, com o uso } \\
\text { da entropia e TOPSIS pelas } \\
\text { variáveis empresa familiar, } \\
\text { pagamento de dividendos, } \\
\text { alavancagem financeira e } \\
\text { ações em tesouraria. }\end{array}$ & $\begin{array}{c}\text { Malmendier e Tate (2005); } \\
\text { Bhandari e Deaves (2006); } \\
\text { Malmendier e Tate (2008); Wong } \\
\text { (2008); Jin e Kothari (2008); } \\
\text { Schrand e Zechman (2012); Hribar } \\
\text { e Yang (2015); Li e Hung (2013); } \\
\text { Banerjee et al. (2014); Hsieh et al. } \\
\text { (2014). }\end{array}$ \\
\hline $\begin{array}{l}\text { Variáveis de } \\
\text { Controle }\end{array}$ & ROA & $\frac{\text { Lucro Líquido }}{\text { Ativo Total }}$ & $\begin{array}{c}\text { Balsam, Haw e Lilien (1995); } \\
\text { Dechow e Dichev (2002); Doyle, } \\
\text { Ge e Mcvay (2007); Barth, } \\
\text { Landsman e Lang (2008); Dechow, } \\
\text { Ge e Schrand (2010) }\end{array}$ \\
\hline
\end{tabular}


Tabela 2 (continuação)

\begin{tabular}{|c|c|c|c|}
\hline Variáveis & Descrição & Métrica & Sustentação teórica \\
\hline \multirow{4}{*}{$\begin{array}{c}\text { Variáveis de } \\
\text { Controle }\end{array}$} & ROE & $\frac{\text { Lucro Líquido }}{\text { Patrimônio Líquido }}$ & $\begin{array}{c}\text { Balsam et al. (1995); Dechow e } \\
\text { Dichev (2002); Doyle et al. (2007); } \\
\text { Barth et al. (2008); Dechow et al. } \\
\text { (2010) }\end{array}$ \\
\hline & Endividamento C.P & $\frac{\text { Passivo Circulante }}{\text { Ativo Total }}$ & $\begin{array}{l}\text { Labelle (1990); Malmquist (1990); } \\
\text { Balsam et al. (1995); Minton e } \\
\text { Schrand (1999) }\end{array}$ \\
\hline & Endividamento L.P & $\frac{\text { Passivo Não Circulante }}{\text { Ativo Total }}$ & $\begin{array}{c}\text { Labelle (1990); Malmquist (1990); } \\
\text { Balsam et al. (1995); Minton e } \\
\text { Schrand (1999) }\end{array}$ \\
\hline & Tamanho & $\begin{array}{l}\text { Logaritmo natural do ativo total } \\
\text { da empresa i no ano } t \text {. }\end{array}$ & $\begin{array}{c}\text { Francis, Khurana e Pereira (2004); } \\
\text { Dechow et al. (2010); } \\
\text { Gaio (2010) }\end{array}$ \\
\hline
\end{tabular}

Nota. Fonte: Elaborada pelos autores.

Observa-se na Tabela 2 que a variável dependente foi composta pelos accruals discricionários, e as variáveis independentes pela alavancagem financeira, volume de ações em tesouraria, controle familiar e volume de pagamento de dividendos. Posteriormente, para teste de robustez, foram adicionadas as variáveis de controle relacionadas ao retorno sobre o ativo, retorno sobre o patrimônio líquido, endividamento de curto e longo prazo e tamanho das organizações. Tais variáveis de controle foram selecionadas por se apresentarem em outros estudos sobre o tema, como incentivos para as práticas de gerenciamento de resultados.

Adicionalmente, as variáveis independentes (ações em tesouraria; volume de pagamento de dividendos; alavancagem financeira; empresa familiar), que serviram de atributos do excesso de confiança dos gestores, foram agregadas em único ranking para determinar o nível geral de excesso de confiança. O ranking agregado foi construído por meio da Technique for Order Preference by Smilarity to Ideal Solution (TOPSIS), com uso da entropia para definição do peso de cada vetor. O TOPSIS é baseado no ranking de alternativas para obter a melhor seleção alternativa, que está próxima da solução ideal, considerada a distância da solução ideal e da solução anti-ideal (Bulgurcu, 2012).

A Figura 1 mostra o modelo KS (1995) utilizado para identificar os accruals discricionários.

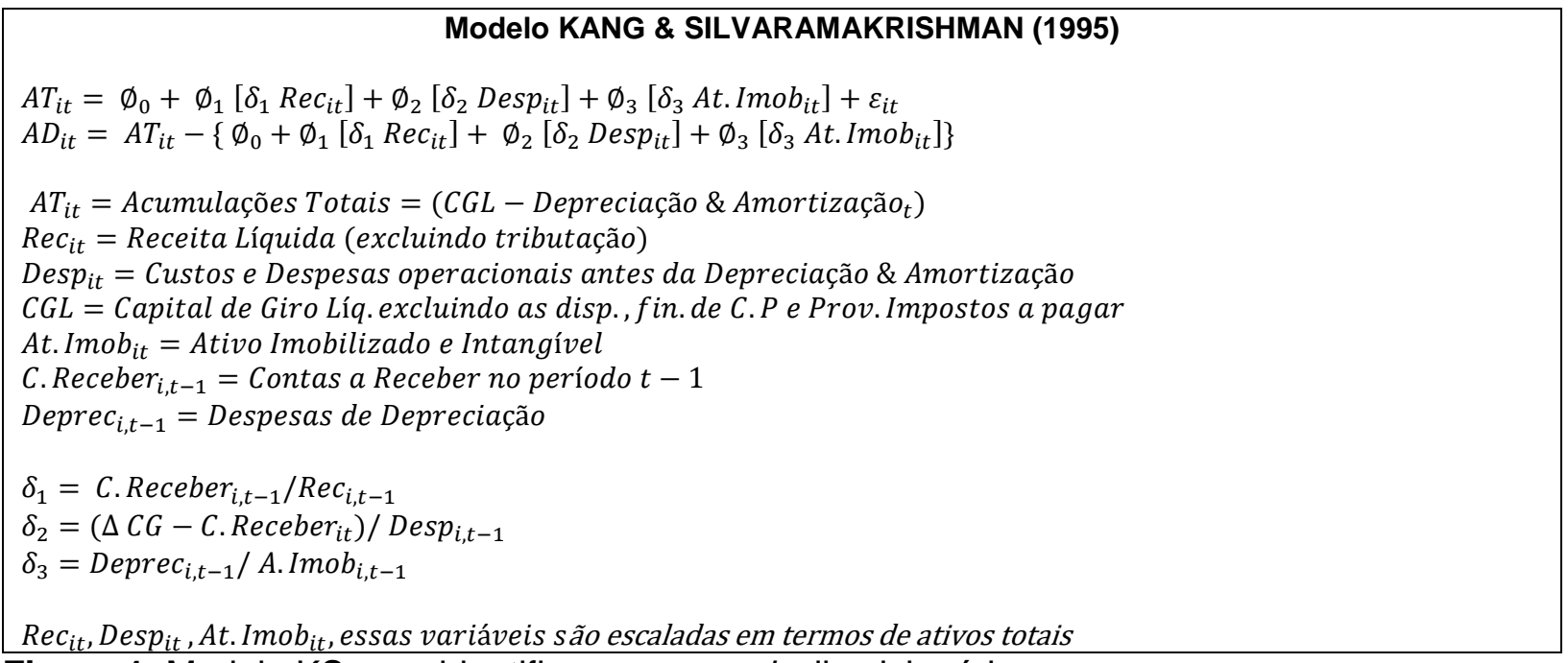

Figura 1. Modelo KS para identificar os accruals discricionários

Fonte: Adaptado de Martinez (2008).

Com os dados do gerenciamento de resultados e com as variáveis independentes foi possível aplicar o modelo de regressão linear com o uso do software SPSS. A partir da aplicação da modelagem foi possível verificar se as variáveis independentes do excesso de confiança exercem influência no nível de gerenciamento de resultados. Tem-se a equação 1 para esboçar o presente modelo de regressão:

$G R=\beta_{1}+\beta_{2} A T+\beta_{3} P D+\beta_{4} A F+\beta_{5} E F+\varepsilon$ 
Em que:

$\mathrm{GR}=$ Accruals Discricionários - Gerenciamento de Resultados;

AT = Ações em Tesouraria;

$\mathrm{PD}=$ Pagamento de Dividendos;

$\mathrm{AF}=$ Alavancagem Financeira;

$\mathrm{EF}=$ Empresa Familiar.

Posteriormente, os atributos (empresa familiar; ações em tesouraria; pagamento de dividendos; alavancagem financeira) foram agrupados em um ranking (índice), por meio do TOPSIS, no intuito de determinar um indicador de excesso de confiança do CEO de cada organização. Estabeleceu-se um modelo de regressão adicional para explicar o nível de gerenciamento de resultados, composto pela variável explicativa ranking do excesso de confiança e pelas variáveis de controle relacionadas ao desempenho, endividamento e tamanho da empresa. O modelo de regressão descrito é representado pela equação 2:

$G R=\beta_{1}+\beta_{2} I E C+\beta_{3} R O A+\beta_{4} R O E+\beta_{5}$ End.C.P $+\beta_{6}$ End.L.P + Tamanho $+\varepsilon$

Em que:

$\mathrm{GR}=$ Accruals Discricionários - Gerenciamento de Resultados;

IEC= índice (ranking) do Excesso de Confiança;

$\mathrm{ROA}=$ Retorno sobre o ativo;

ROE = Retorno sobre o Patrimônio Líquido;

End. C.P = Endividamento de Curto Prazo;

End. L.P = Endividamento de Longo Prazo;

Tamanho $=$ Logaritmo natural do ativo total.

A Figura 2 mostra como foi elaborado o agrupamento das variáveis numa única variável para medir o excesso de confiança de gestores, bem como o modelo preliminar de teste do excesso de confiança com relação à prática de gerenciamento de resultados.

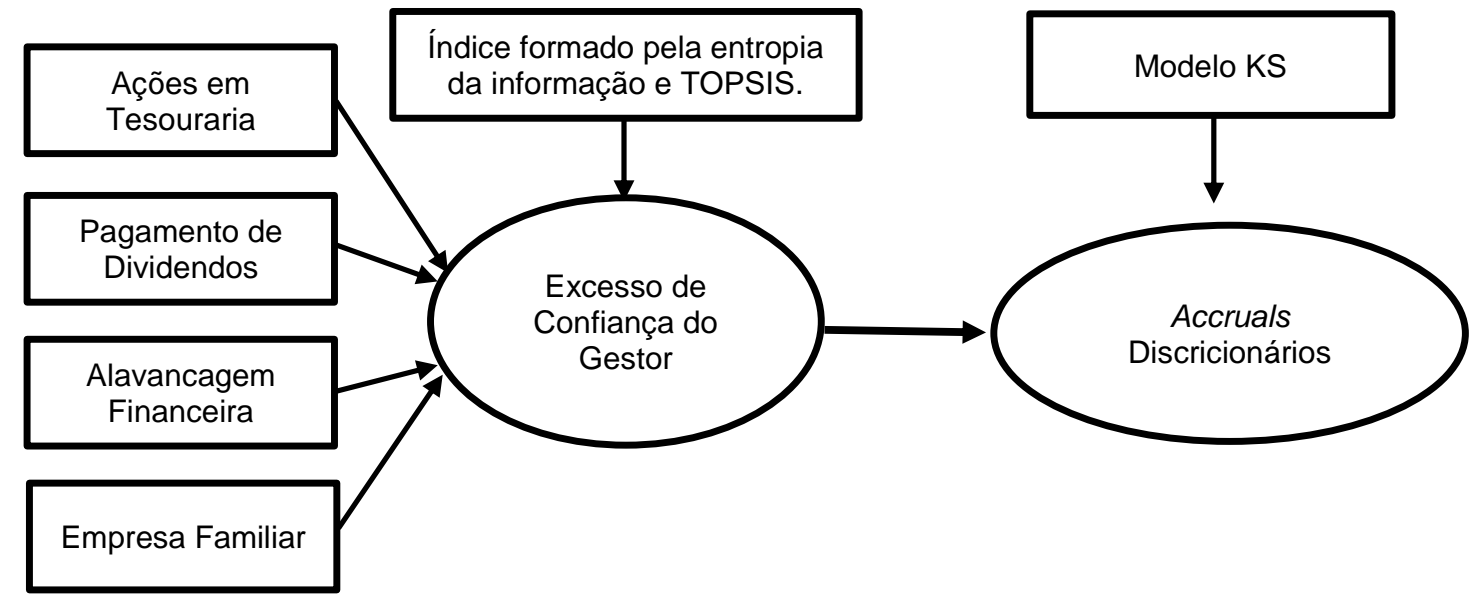

Figura 2. Modelo para explicar a construção da variável excesso de confiança e sua relação com gerenciamento de resultados

Fonte: Elaborado pelos autores.

\section{APRESENTAÇÃO E ANÁLISE DOS RESULTADOS}

Este tópico apresenta os achados encontrados mediante a aplicação dos métodos estabelecidos na metodologia da pesquisa, com vistas a atender ao objetivo proposto pelo estudo. Nesse sentido, a Tabela 3 apresenta a estatística descritiva das variáveis utilizadas na pesquisa. 
Tabela 3

\section{Estatística descritiva}

\begin{tabular}{lcccc}
\hline \multicolumn{1}{c}{ Variável } & Mínimo & Máximo & Média & Desvio Padrão \\
\hline Accruals Discricionários & $-3,8281$ & 2,2083 & 0,000 & 0,8944 \\
Ações em Tesouraria & 0,0000 & $2.104 .524,00$ & $41.089,70$ & $198.062,58$ \\
Pagamento de Dividendos & 0,0000 & $249.037 .996,00$ & $4.811 .945,63$ & $22.465 .880,41$ \\
Alavancagem Financeira & 0,0061 & 0,6074 & 0,2995 & 0,1519 \\
Empresa familiar & 0,0000 & 1,0000 & 0,4803 & 0,5016 \\
ROA - Retorno sobre Ativo & $-0,124$ & 0,558 & 0,048 & 0,071 \\
ROE - Retorno sobre PL & $-1,250$ & 0,805 & 0,090 & 0,227 \\
End. C.P & 0,014 & 0,623 & 0,212 & 0,124 \\
End. L.P & 0,000 & 0,705 & 0,330 & 0,149 \\
Tamanho (Ativo Total) & 48.601 & 752.966 .638 & 17.181 .136 & 68.488 .989 \\
\hline
\end{tabular}

Nota. Fonte: Dados da pesquisa.

Verifica-se na Tabela 3 que o mínimo dos accruals discricionários foi de $-3,8281$ e o máximo de 2,2083, com desvio padrão de 0,8944. Em relação à variável de ações em tesouraria, o mínimo foi de 0,000 pela existência de empresas que não mantinham ações em tesouraria, e o máximo de $\mathrm{R} \$ 2.104 .524,00$. Em relação ao pagamento de dividendos, verifica-se que há empresas que não possuem pagamento de dividendos no período, conforme o mínimo de 0,00. Já o valor máximo de pagamento de dividendos foi de $R \$ 249.037 .996,00$.

Em relação à alavancagem financeira verifica-se que a média foi de 0,2995, e o máximo de 0,6074. Em relação às empresas familiares foi verificado que das 127 empresas estudadas, 61 são familiares e 66 não são familiares. Portanto, o percentual de empresas familiares foi de $48,03 \%$. Com relação às variáveis de controle, observa-se que em médias empresas apresentam um retorno sobre o ativo de 0,048 , retorno sobre o patrimônio líquido de 0,090, endividamento de curto prazo de 0,212, endividamento de longo prazo de 0,330 e tamanho das empresas pelo total de ativos de $\mathrm{R} \$ 17.181 .136,00$.

Destaca-se a alta variabilidade nos dados, o que pode comprometer a estimativa dos parâmetros, já que se estima pela média. Posteriormente, foram aplicadas as regressões propostas para responder às hipóteses apresentadas na construção teórica. Adicionalmente, com as variáveis expostas na Tabela 3, foram comprovados, estatisticamente, os pressupostos de normalidade, ausência de autocorrelação, heterocedasticidade, multicolinearidade e linearidade.

A Tabela 4 mostra o resumo do modelo de regressão linear das variáveis independentes compreendidas pelas ações em tesouraria, pagamento de dividendos, alavancagem contábil e empresa familiar com relação à variável dependente dos accruals discricionários.

Tabela 4

Regressão das variáveis de mensuração do excesso de confiança com relação aos accruals discricionários

\begin{tabular}{|c|c|c|c|c|c|c|c|}
\hline Modelo & $\begin{array}{c}\begin{array}{c}\text { Coeficientes não } \\
\text { padronizados }\end{array} \\
\text { B }\end{array}$ & $\mathbf{T}$ & Sig. & VIF & $\begin{array}{c}\text { Modelo } \\
\mathbf{R}^{\mathbf{2}}\end{array}$ & $\begin{array}{l}\text { Durbin } \\
\text { Watson }\end{array}$ & $\begin{array}{c}\text { Modelo } \\
\text { Sig. }\end{array}$ \\
\hline (Constante) & 0,578 & 2,382 & $0,019^{*}$ & & & & \\
\hline Ações Tesouraria & $-0,044$ & $-2,887$ & $0,005^{*}$ & 1,099 & & & \\
\hline Pagamento Dividendos & 0,035 & 1,770 & 0,079 ** & 1,082 & 0,194 & 1,660 & $0,000^{*}$ \\
\hline Alavancagem Contábil & $-0,007$ & $-3,573$ & $0,001^{*}$ & 1,052 & & & \\
\hline Empresa Familiar & 0,009 & 0,059 & 0,953 & 1,066 & & & \\
\hline
\end{tabular}

Nota. * significância ao nível de $5 \%$

** significância ao nível de $10 \%$

Fonte: Dados da pesquisa.

Verifica-se na Tabela 4 que 19,40\% da variabilidade total do gerenciamento de resultados (accruals discricionários) pode ser explicada pelas ações em tesouraria, pagamento de dividendos, alavancagem financeira e empresa familiar. Conforme a base teórica, as variáveis podem representar o comportamento de excesso de confiança dos gestores. Ocorrem, assim, evidências de uma possível influência do excesso de confiança dos CEOs sobre o nível de gerenciamento de resultados. 
O modelo de regressão apresentou significância ao nível de $5 \%$, com p-value de 0,000. É possível comprovar que $19,40 \%$ da variabilidade dos accruals discricionários pode ser explicada pelo excesso de confiança de gestores, medido pelas variáveis individuais de ações em tesouraria, pagamento de dividendos, alavancagem financeira e empresa familiar.

Os coeficientes do modelo de regressão comprovam que o maior volume de ações em tesouraria influencia negativamente a prática oportunista de gerenciamento de resultados para aumentar os lucros, ao nível de significância de $5 \%(0,005)$. O achado não corrobora com a literatura, a qual expressa que quanto maior o volume de ações em tesouraria, maior o excesso de confiança, e, por conseguinte, maior o nível de gerenciamento de resultados para aumentar os lucros, rejeitando a $\mathrm{H} 1 \mathrm{~d}$.

Em relação ao pagamento de dividendos, a literatura expressa que o menor volume de pagamento de dividendos representa maior o excesso de confiança dos gestores. Desse modo, verifica-se que o resultado foi inverso, o maior pagamento de dividendos (gestores com menor excesso de confiança) influencia no maior nível de gerenciamento de resultados para aumentar os lucros, com nível de significância de 10\%, adverso à H1b.

Complementarmente, a literatura expressa que a maior alavancagem financeira é atributo para estabelecer o maior excesso de confiança dos gestores e, portanto, os achados demonstram que a menor alavancagem (menor excesso de confiança dos gestores) influencia positivamente no nível de gerenciamento de resultados para aumentar os lucros, inverso à $\mathrm{H} 1 \mathrm{c}$.

Por fim, os resultados indicam que a empresa familiar não apresenta significância de impacto no nível de gerenciamento de resultados, não possibilita exercer inferência sobre a H1a. Os achados contrariam as hipóteses prévias. Contudo, demonstram evidências importantes de que os CEOs com menor excesso de confiança tendem a exercer práticas oportunistas de gerenciamento de resultados para aumentar os lucros. A contradição nas hipóteses pode ter ocorrido pelo fato de a base teórica ser de países com mercados acionários desenvolvidos e com forte legislação de auditoria e governança corporativa, como é o caso de países em que as empresas são monitoradas com a Lei Sarbanes-Orley. A Tabela 5 demonstra um resumo dos pressupostos das sub-hipóteses e respetivos achados.

Tabela 5

Resultados da pesquisa em relação à construção das sub-hipóteses

\begin{tabular}{ccc}
\hline $\begin{array}{c}\text { Resultados das variáveis de } \\
\text { excesso de confiança }\end{array}$ & $\begin{array}{c}\text { Resultados conforme atributos } \\
\text { do Excesso de Confiança }\end{array}$ & $\begin{array}{c}\text { Resultados com a variável } \\
\text { dependente }\end{array}$ \\
\hline - Ações em Tesouraria & - Excesso de Confiança & + Gerenciamento de Resultados \\
+ Pagamento Dividendos & - Excesso de Confiança & + Gerenciamento de Resultados \\
- Alavancagem Contábil & - Excesso de Confiança & + Gerenciamento de resultados \\
\hline Nota. Fonte: Elaborada pelos autores. & &
\end{tabular}

Verifica-se na Tabela 5 que os resultados foram inversos aos apontados pela literatura. Tais resultados sugerem que o menor excesso de confiança, medido pelos atributos de menor volume de ações em tesouraria, maior volume de pagamento de dividendos e menor alavancagem financeira, influenciam na prática oportunista de gerenciamento de resultados para aumentar os lucros. Infere-se que os achados podem ter sofrido interferência das taxas de juros e políticas de crédito do mercado brasileiro, no caso dos fatores relacionados à alavancagem e a dividendos. Com relação ao volume de ações em tesouraria e às empresas familiares, os resultados podem ter sofrido interferência da cultura acionária, bem como da grande proporção de empresas familiares que compõe o mercado brasileiro.

Os fatores apresentados são totalmente contrários aos encontrados no mercado dos Estados Unidos e do Taiwan, e, portanto, trazem resultados complementares para as pesquisas em mercados com características similares aos do ambiente brasileiro. A Tabela 6 mostra o modelo de regressão com o uso do índice de excesso de confiança e a inclusão das variáveis de controle no impacto sobre os accruals discricionários. 
Tabela 6

Coeficientes do modelo de regressão com uso do índice de excesso de confiança

\begin{tabular}{|c|c|c|c|c|c|c|c|}
\hline Modelo & $\begin{array}{c}\begin{array}{c}\text { Coeficientes não } \\
\text { padronizados }\end{array} \\
\text { B }\end{array}$ & $\mathbf{T}$ & Sig. & VIF & $\begin{array}{c}\text { Modelo } \\
\mathbf{R}^{2}\end{array}$ & $\begin{array}{l}\text { Durbin } \\
\text { Watson }\end{array}$ & $\begin{array}{l}\text { Modelo } \\
\text { Sig. }\end{array}$ \\
\hline (Constante) & 1,290 & 1,728 & $0,087^{* *}$ & & \multirow{7}{*}{0,417} & \multirow{7}{*}{1,664} & \multirow{7}{*}{$0,000^{*}$} \\
\hline Excesso de Confiança & $-2,610$ & $-3,963$ & $0,000^{*}$ & 1,286 & & & \\
\hline ROA & $-2,012$ & $-1,405$ & 0,163 & 2,673 & & & \\
\hline ROE & 0,286 & 0,635 & 0,527 & 2,690 & & & \\
\hline End. C.P & $-4,056$ & $-7,734$ & $0,000^{*}$ & 1,086 & & & \\
\hline End. L.P & $-0,189$ & $-0,390$ & 0,697 & 1,353 & & & \\
\hline Tamanho & 0,068 & 0,611 & 0,542 & 1,252 & & & \\
\hline
\end{tabular}

Nota. * significância ao nível de $5 \%$

** significância ao nível de $10 \%$

Fonte: Dados da pesquisa.

Observa-se na Tabela 6 que $41,70 \%$ da variabilidade total do gerenciamento de resultados pode ser explicada pelo índice de excesso de confiança e pelas variáveis de controle. Confirma-se a evidência da análise preliminar, de que o excesso de confiança influencia no gerenciamento de resultados. Além disso, o modelo de regressão apresentou significância ao nível de $5 \%$. Comprovam que o excesso de confiança e as variáveis de controle podem explicar a prática de gerenciamento de resultados nas organizações estudadas.

Os resultados reforçam as evidências apresentadas na Tabela 1 (primeira modelagem), de que o índice de excesso de confiança apresentou influência negativa sobre o gerenciamento de resultados. Pode ser confirmado que o menor excesso de confiança de gestores influencia na prática oportunista de gerenciamento de resultados para aumentar os lucros. Os resultados confirmam as evidências de Schrand e Zechman (2012) sobre a possível influência do excesso de confiança de gestores no gerenciamento de resultados. Por outro lado, os resultados contrariam as evidências de Hribar e Yang (2015) Li e Hung (2013) e Hsieh et al. (2014), de que o maior excesso de confiança de gestores influenciaria na prática oportunista de gerenciamento dos resultados para aumentar os lucros.

Pressupõe-se que a divergência em relação aos estudos anteriores pode decorrer da insegurança dos gestores sobre o mercado acionário brasileiro, visto que as pesquisas sobre o tema foram realizadas em países desenvolvidos, com mercado acionário consolidado e confiável a investidores. Gestores com menor excesso de confiança tenderiam a práticas oportunistas de gerenciamento de resultados, pela insegurança em apresentar resultados contábeis que não satisfaçam às expectativas dos analistas de mercado, acionistas e investidores. Resultados insatisfatórios poderiam causar instabilidade organizacional e perda de reputação dos gestores, fazendo com que aqueles que tenham menor excesso de confiança façam proveito da discricionariedade nas escolhas contábeis.

A contradição das hipóteses contribuiu por maneira para a literatura. Demonstra efeitos inversos do excesso de confiança na qualidade da informação contábil em mercado emergente, com menores níveis de desenvolvimento do mercado de capitais, bem como menor efetividade de atuação dos mecanismos de governança corporativa.

Por fim, com relação às variáveis de controle, foi possível inferir que o menor endividamento de curto prazo provoca maior prática de gerenciamento de resultados mediante escolhas contábeis, o que contraria as evidências de Labelle (1990), Malmquist (1990) e Balsam et al. (1995), de que os maiores níveis de endividamento estariam associados a escolhas contábeis oportunistas para aumentar os lucros.

Além disso, os achados indicam que o retorno sobre ativos e retorno sobre o patrimônio líquido não impactam no nível de gerenciamento de resultados. Tal evidência não confirma exposições de Balsam et al. (1995), Doyle et al. (2007), de que o baixo desempenho organizacional pode oferecer incentivos às práticas oportunistas de gerenciamento de resultados. Por fim, o endividamento de longo prazo e tamanho das organizações não apresentaram influência sobre as práticas oportunistas de gerenciamento de resultados. 


\section{CONCLUSÕES}

O objetivo do estudo foi identificar a influência do excesso de confiança de gestores no gerenciamento de resultados nas empresas brasileiras. Inicialmente verificou-se que empresas com ações em tesouraria apresentaram menor gerenciamento de resultados, o que permite inferir que o menor excesso de confiança, caracterizado por menores valores em ações em tesouraria, influencia no maior nível de gerenciamento de resultados.

Conclusões compatíveis podem ser obtidas, de que o maior pagamento de dividendos (caracterizado pela literatura como gestores com menor excesso de confiança) apresentou impacto positivo no gerenciamento de resultados. Desse modo, a maior remuneração por meio de dividendos apresenta relação positiva sobre o gerenciamento de resultados. Mostra que empresas com gestores menos confiantes, que distribuem resultados e não deixam reaplicados na organização, apresentam práticas oportunistas de gerenciamento de resultados.

Os achados sugerem que a empresa familiar não apresenta relação com o nível de gerenciamento de resultados. Não podem ser inferidas, nesse aspecto, conclusões acerca do excesso de confiança de gestores. E, por fim, a menor alavancagem financeira apresentou influência positiva sobre o gerenciamento de resultados. Sugere que o menor excesso de confiança tem efeito positivo sobre esse gerenciamento. De modo geral, sugere-se que as variáveis determinadas pela literatura, para medir o excesso de confiança, apresentaram influência sobre a prática oportunista de gerenciamento de resultados, embora os resultados tenham sido controversos aos apontados pela literatura (Malmendier \& Tate, 2008; Jin \& Kothari, 2008; Hribar \& Yang, 2015; Li \& Hung, 2013; Hsieh et al., 2014; Banerjee et al. 2014).

A consistência para os achados foi obtida pelo ranking do excesso de confiança, de que os resultados reforçam que o menor excesso de confiança influencia no aumento das práticas oportunistas de gerenciamento de resultados para aumentar os lucros. Argumenta-se que as diferenças encontradas podem ser pautadas nas características do mercado financeiro brasileiro, apresentam níveis diferenciados sobre o volume de ações em tesouraria, na alavancagem financeira, no pagamento de dividendos e em percentual de empresas com controle familiar.

O resultado divergente para o contexto brasileiro, em relação aos achados internacionais, pode ser explicado pelo argumento de que os gestores com menor confiança podem estar fazendo uso do gerenciamento de resultados para demonstrar um resultado financeiro que aumente sua reputação perante o mercado de capitais e para a própria organização. Além disso, os gestores com menor confiança presumem que suas habilidades são inferiores às dos concorrentes e, portanto, utilizam do gerenciamento de resultados para atender às expectativas que foram prospectadas, satisfazendo sua autoestima. O fato corrobora o estudo de Lin et al. (2005), de que os aspectos comportamentais dos indivíduos podem ser úteis para explicar suas escolhas financeiras.

Os estudos de Malmendier e Tate (2008), Jin e Kothari (2008), Hribar e Yang (2015), Li e Hung (2013), Hsieh et al. (2014) e Banerjee et al. (2014), com gestores de empresas norte americanas, sugerem que o maior excesso de confiança causa maior gerenciamento de resultados. Nesse sentido, as empresas norte-americanas são monitoradas pela Lei SarbanesOxley, que pode estar restringindo os gestores, com menor excesso de confiança, a efetuarem o gerenciamento de resultados, por medo de serem descobertos. Por outro lado, os gestores excessivamente confiantes não ficariam intimidados com mecanismos de monitoramento mais rígidos e agiriam de acordo com seus interesses. Os fatores expostos podem oferecer argumentos para os resultados controversos encontrados no contexto brasileiro, e portanto, fatores culturais, pessoais e macroeconômicos são muito específicos de cada país. Impactam também, no comportamento dos indivíduos.

Por fim, conclui-se que a psicologia individual pode afetar as escolhas contábeis oportunistas (Hribar \& Yang, 2015). Contribui para uma maior integração entre os aspectos comportamentais dos gestores (CEOs) e as práticas oportunistas de gerenciamento de resultados para aumentar os lucros. Adicionalmente, os achados demonstraram que os analistas, investidores e demais stakeholders devem observar o comportamento dos agentes corporativos na avaliação dos resultados reportados pelas firmas, sem deixar de considerar as características do mercado acionário, auditoria, governança corporativa e, principalmente, os aspectos culturais para investimentos no mercado de capitais. 
Recomenda-se pesquisas futuras com aplicação de questionários que captem o excesso de confiança dos gestores, diretores e executivos das organizações. Ademais, é importante a relação de pesquisas que considerem o excesso de confiança sobre outras perspectivas (premissas) da qualidade da informação contábil (conservadorismo, tempestividade, relevance value, persistência, dentre outras). Por fim, podem ser utilizadas variáveis adicionais para expressar o excesso de confiança dos CEOs, como a idade, a escolaridade e o tempo de mandato.

\section{REFERÊNCIAS}

Ahmed, A. S., \& Duellman, S. (2013). Managerial overconfidence and accounting conservatism. Journal of Accounting Research, 51(1), 1-30.

Alicke, M. D., Klotz, M. L., Breitenbecher, D. L., Yurak, T. J., \& Vredenburg, D. S. (1995). Personal contact, individuation, and the better-than-average effect. Journal of Personality and Social Psychology, 68(5), 804.

Anderson, R. C., \& Reeb, D. M. (2003). Founding-family ownership and firm performance: evidence from the S\&P 500. The Journal of Finance, 58(3), 1301-1328.

Andriosopoulos, D., Andriosopoulos, K., \& Hoque, H. (2013). Information disclosure, CEO overconfidence, and share buyback completion rates. Journal of Banking \& Finance, 37(12), 5486-5499.

Baker, M., Ruback, R. S., \& Wurgler, J. (2004). Behavioral corporate finance: A survey (No. w10863). National Bureau of Economic Research.

Balsam, S., Haw, I. M., \& Lilien, S. B. (1995). Mandated accounting changes and managerial discretion. Journal of Accounting and Economics, 20(1), 3-29.

Banerjee, S., Humphery-Jenner, M., \& Nanda, V. (2013). Harnessing CEO overconfidence. UNSW Australian School of Business Research Paper.

Barber, B. M., \& Odean, T. (2001). Boys will be boys: Gender, overconfidence, and common stock investment. Quarterly Journal of Economics, 261-292.

Barros, L. A. B. D. C., \& Da Silveira, A. D. M. (2009). Overconfidence, managerial optimism and the determinants of capital structure. Brazilian Review Finance, 6(3), 293-335.

Barth, M. E., Landsman, W. R., \& Lang, M. H. (2008). International accounting standards and accounting quality. Journal of Accounting Research, 46(3), 467-498.

Ben-David, I., Graham, J. R., \& Harvey, C. R. (2007). Managerial overconfidence and corporate policies (No. w13711). National Bureau of Economic Research.

Bulgurcu, B. K. (2012). Application of TOPSIS technique for financial performance evaluation of technology firms in Istanbul stock exchange market. Procedia-Social and Behavioral Sciences, 62(24), 1033-1040.

Cooper, A. C., Woo, C. Y., \& Dunkelberg, W. C. (1988). Entrepreneurs' perceived chances for success. Journal of Business Venturing, 3(2), 97-108.

Dalton, D. R., \& Daily, C. M. (2001). Director stock compensation: an invitation to a conspicuous conflict of interests?. Business Ethics Quarterly, 11(01), 89-108.

De Bondt, W. F., \& Thaler, R. H. (1995). Financial decision-making in markets and firms: A behavioral perspective. Handbooks in operations research and management science, 9, 385-410.

Dechow, P. M., \& Dichev, I. D. (2002). The quality of accruals and earnings: The role of accrual estimation errors. The Accounting Review, 77(s-1), 35-59. 
Dechow, P., Ge, W., \& Schrand, C. (2010). Understanding earnings quality: A review of the proxies, their determinants and their consequences. Journal of Accounting and Economics, 50(2), 344-401.

Deshmukh, S., Goel, A. M., \& Howe, K. M. (2013). CEO overconfidence and dividend policy. Journal of Financial Intermediation, 22(3), 440-463.

Dittrich, D. A., Güth, W., \& Maciejovsky, B. (2005). Overconfidence in investment decisions: An experimental approach. The European Journal of Finance, 11(6), 471-491.

Doyle, J. T., Ge, W., \& McVay, S. (2007). Accruals quality and internal control over financial reporting. The Accounting Review, 82(5), 1141-1170.

Dushnitsky, G. (2010). Entrepreneurial optimism in the market for technological inventions. Organization Science, 21(1), 150-167.

Francis, J. R., Khurana, I. K., \& Pereira, R. (2004). The role of accounting and auditing in corporate governance and the development of financial markets around the world. AsiaPacific Journal of Accounting \& Economics, 10(1), 1-30.

Gaio, C. (2010). The relative importance of firm and country characteristics for earnings quality around the world. European Accounting Review, 19(4), 693-738.

Gómez-Mejía, L. R., Haynes, K. T., Núñez-Nickel, M., Jacobson, K. L., \& Moyano-Fuentes, J. (2007). Socioemotional wealth and business risks in family-controlled firms: Evidence from Spanish olive oil mills. Administrative Science Quarterly, 52(1), 106-137.

Heaton, J. B. (2002). Managerial optimism and corporate finance. Financial management, 33-45.

Hilary, G., \& Hsu, C. (2011). Endogenous overconfidence in managerial forecasts. Journal of Accounting and Economics, 51(3), 300-313.

Hmieleski, K. M., \& Baron, R. A. (2009). Entrepreneurs' optimism and new venture performance: A social cognitive perspective. Academy of Management Journal, 52(3), 473-488.

Hribar, P., Yang, H. (2015). CEO overconfidence and management forecasting. Contemporary Accounting Research, 33(1), 204-227.

Hsieh, T. S., Bedard, J. C., \& Johnstone, K. M. (2014). CEO overconfidence and earnings management during shifting regulatory regimes. Journal of Business Finance \& Accounting, 41(9-10), 1243-1268.

Simon, M., \& Houghton, S. M. (2003). The relationship between overconfidence and the introduction of risky products: Evidence from a field study. Academy of Management Journal, 46(2), 139-149.

Jin, L., \& Kothari, S. P. (2008). Effect of personal taxes on managers' decisions to sell their stock. Journal of Accounting and Economics, 46(1), 23-46.

Kang, S. H., \& Sivaramakrishnan, K. (1995). Issues in testing earnings management and an instrumental variable approach. Journal of Accounting Research, 33(2), 353-367.

Ko, K. J., \& Huang, Z. J. (2007). Arrogance can be a virtue: Overconfidence, information acquisition, and market efficiency. Journal of Financial Economics, 84(2), 529-560.

Labelle, R. (1990). Bond covenants and changes in accounting policy: Canadian evidence. Contemporary Accounting Research, 6(2), 677-698.

Landier, A., \& Thesmar, D. (2009). Financial contracting with optimistic entrepreneurs. Review of Financial Studies, 22(1), 117-150.

Lee, J. M. M., Hwang, B. H., \& Chen, H. (2014). Behavioral Differences Between Founder CEOs and Professional CEOs: The Role of Overconfidence. In Academy of Management Proceedings, 1, 17214, Academy of Management. 
Li, I. C., \& Hung, J. H. (2013). The moderating effects of family control on the relation between managerial overconfidence and earnings management. Review of Pacific Basin Financial Markets and Policies, 16(02).

Lin, Y. H., Hu, S. Y., \& Chen, M. S. (2005). Managerial optimism and corporate investment: Some empirical evidence from Taiwan. Pacific-Basin Finance Journal, 13(5), 523-546.

Lowe, R. A., \& Ziedonis, A. A. (2006). Overoptimism and the performance of entrepreneurial firms. Management Science, 52(2), 173-186.

Malmendier, U., \& Tate, G. (2005). CEO overconfidence and corporate investment. The Journal of Finance, 60(6), 2661-2700.

Malmendier, U., \& Tate, G. (2008). Who makes acquisitions? CEO overconfidence and the market's reaction. Journal of Financial Economics, 89(1), 20-43.

Malmendier, U., Tate, G., \& Yan, J. (2011). Overconfidence and early-life experiences: the effect of managerial traits on corporate financial policies. The Journal of Finance, 66(5), 16871733.

Malmquist, D. H. (1990). Efficient contracting and the choice of accounting method in the oil and gas industry. Journal of Accounting and Economics, 12(1), 173-205.

Martinez, A. L. (2008). Detectando Earnings Management no Brasil: Estimando os Accruals Discricionários. Revista de Contabilidade e Finanças da USP, 19(46), 7-17.

Martinez, A. L. (2013). Gerenciamento de resultados no Brasil: um survey da literatura. BBRBrazilian Business Review, 10(4), 1-31.

Matsumoto, A. S., \& Parreira, E. M. (2009). Uma pesquisa sobre o Gerenciamento de Resultados Contábeis: causas e conseqüências. Contabilidade, Gestão e Governança, 10(1).

Miller, D., Breton-Miller, L., \& Scholnick, B. (2008). Stewardship vs. stagnation: An empirical comparison of small family and non-family businesses. Journal of Management Studies, 45(1), 51-78.

Minton, B. A., \& Schrand, C. (1999). The impact of cash flow volatility on discretionary investment and the costs of debt and equity financing. Journal of Financial Economics, 54(3), 423-460.

Nelson, T. (2003). The persistence of founder influence: Management, ownership, and performance effects at initial public offering. Strategic Management Journal, 24(8), 707724.

Peterson, R. L. (2007). Inside the Investor's Brain, Wiley Trading, Hoboken, NJ.

Russo, J. E., \& Schoemaker, P. J. (1992). Managing overconfidence. Sloan Management Review, 33(2), 7-17.

Santos, A. D., \& Grateron, I. R. G. (2003). Contabilidade criativa e responsabilidade dos auditores. Revista Contabilidade \& Finanças, 14(32), 07-22.

Schrand, C. M., \& Zechman, S. L. (2012). Executive overconfidence and the slippery slope to financial misreporting. Journal of Accounting and Economics, 53(1), 311-329.

Svenson, O. (1981). Are we all less risky and more skillful than our fellow drivers?. Acta Psychologica, 47(2), 143-148.

Taylor, S. E., \& Brown, J. D. (1988). Illusion and well-being: a social psychological perspective on mental health. Psychological Bulletin, 103(2), 193.

Thaler, R. H. (1999). The End of Behavioral Finance. Financial Analysts Journal, 55(6), 12-17.

Watts, R. L., \& Zimmerman, J. L. (1990). Positive accounting theory: a ten-year perspective. Accounting Review, 65(1), 131-156.

Weinstein, N. D. (1980). Unrealistic optimism about future life events. Journal of Personality and Social Psychology, 39(5), 806. 